C. THEORY RELEVANT TO UV SPECTRA 


\title{
A DISCUSSION OF THE THEORY FOR INTERPRETING ULTRAVIOLET STELLAR SPECTRA
}

\author{
ANNE B. UNDERHILL \\ The Astronomical Institute, Utrecht, The Netherlands
}

\begin{abstract}
The observed spectral distribution from a star will, in principle, give information about the parts of the atmosphere from which the radiation comes. Most detailed theoretical studies assume that the atmosphere may be represented by plane parallel layers, but in the case of very strong absorption lines and weak continuous absorption, a case which is encountered in the ultraviolet spectral region of $B$ stars, the radiation received from the centers of the lines may come from an entirely different part of the star than does that from the far wings of the lines or from the continuous spectrum. In the case of strong lines, because stars are three dimensional objects and because a field of motion may occur in the outermost part of the atmosphere, the appearance of the ultraviolet line spectrum may be poorly predicted using the hypothesis of stationary plane parallel layers.

Some numerical examples are presented demonstrating that with the UV resonance lines the opacity in the centre of a line may exceed the continuous opacity by a factor $10^{6}-10^{8}$. A summary is given of the chief factors which should be taken into account in any theory of line formation when the hypothesis of LTE is not valid. A detailed description of the interactions between radiation and matter which can occur is necessary. Some examples of the distribution in energy of the lower energy levels of the ion are presented for typical ions of interest. This distribution and the presence of metastable levels are important factors in determining the significance of non-LTE physics. The paper concludes with remarks indicating that ultraviolet spectra of stars should be obtained with a spectral purity of at east $0.1 \AA$ if progress is to be made in understanding the physical state of stars.
\end{abstract}

\section{Introduction}

Information about the physical conditions in stellar atmospheres is found by comparing observed stellar spectra with spectra predicted using a model of the stellar atmosphere and a theory of the process of line formation. The model is usually specified by its composition, effective temperature and $\log g$. The shape and strengths of the predicted line profiles depend upon the assumed abundance of the carrier atom (ion) and the type of expression used to represent the interactions between radiation and matter which lead to the formation of the stellar absorption or emission lines. When satisfactory agreement has been obtained between predicted and observed profiles and line strengths, the star is said to be like the model. There is no guarantee that a model which explains some spectroscopic details successfully will explain the full spectrum of the star.

It is convenient to divide the UV spectral region of B stars into two parts:

(i) The far ultraviolet which lies between $911.6 \AA$ (the Lyman limit) and $1900 \AA$.

(ii) The near ultraviolet which lies between $1900 \AA$ and $3100 \AA$, the effective ozone cut-off.

Many resonance lines and strong lines from low-lying levels in the first, second, third and fourth spectra of the light elements occur in the far ultraviolet region and here the continuous opacity due to $\mathrm{H}, \mathrm{He}$ I and $\mathrm{He}$ II is significantly smaller than that 
in the blue-violet spectral region. The near ultraviolet region contains the resonance lines of MgII and FeII and it is full of lines, mostly subordinate, from the second and third spectra of the metals and light elements. Here the continuous opacity is about the same as in the normally observed blue-violet spectral region. This means that a model which gives a satisfactory representation of the continuous spectrum in the blue-violet region will also represent well the continuous spectrum in the near ultraviolet region. The only spectra from relatively abundant atoms which do not have resonance lines at wavelengths longer than $911.6 \AA$ are HeI, He II, OII, OIII, NeI and Ne II. It is expected that interstellar absorption in the Lyman continuum will prevent observation of the spectra of B stars at wavelengths shorter than $911.6 \AA$.

\section{Geometric Considerations}

The simplest configuration is plane parallel layers in hydrostatic equilibrium which is the configuration for which most detailed studies have been made. The examples quoted here are for this case.

The part of the atmosphere most important for determining the value of the emergent flux $F_{v}$ at any frequency is that lying outside the geometric level $z_{1}$, where

$$
t_{v}=-\int_{z_{1}}^{\infty}\left(\kappa_{v}+l_{v}+\sigma\right) \varrho \mathrm{d} z \approx 0.4 .
$$

This is an empirical relationship which can be derived from the quadrature formulas used to calculate the emergent flux from model atmospheres when spectrum formation takes place as though LTE exists. In this expression the geometric coordinate is measured outward from the center of the star, the total continuous absorption coefficient at frequency $v$ is represented by $\kappa_{v}$, the total absorption coefficient due to line sources of opacity by $l_{v}$, and the electron scattering coefficient by $\sigma$. These coefficients are per unit mass; the density is represented by $\varrho$. At far ultraviolet wavelengths the continuous opacity $\kappa_{v}$ is small and $z_{1}$ will lie deep in the atmosphere for fluxes in the continuum. On the other hand, near the centre of strong lines $l_{v}$ is very large and $z_{1}$ will lie at a high level in the atmosphere.

These considerations give one a feeling for which layers of a model atmosphere one observes when one studies the emergent spectrum in different wavelengths. From the characteristics of the model one can estimate the particle density and electron temperature in the significant layers for each spectral feature and from these quantities one can check whether the exploratory hypothesis of LTE is likely to be valid for understanding the spectral details in question. To demonstrate what is involved, Table I gives the monochromatic optical depth at the centers of the C II, C III and CIV lines expected to be strong, at the centre of $\mathrm{H} \gamma$ and in the continuous spectrum at $1458 \AA$ and at $4340.5 \AA$ for layers in three model atmospheres at which the characteristic optical depth, $\tau$, is $0.001,0.01,0.10$ and 0.50 . The selected models represent fairly well main-sequence stars of types B6, B1.5 and O9 in so far as the normally observed con- 
TABLE I

Monochromatic optical depths in the centres of some lines and in the continuum

\begin{tabular}{|c|c|c|c|c|c|c|c|}
\hline \multirow{2}{*}{$\begin{array}{l}\tau \\
0.001\end{array}$} & Wavelength $(\AA)$ & \multicolumn{2}{|c|}{$B 6 V^{(a)}$} & \multicolumn{2}{|c|}{$\mathrm{B} 1.5 \mathrm{~V}^{(\mathrm{b})}$} & \multicolumn{2}{|c|}{ O9 $\mathrm{V}^{(\mathrm{c})}$} \\
\hline & CII $\quad 1335.3$ & .2255 & +5 & - & & .4040 & +1 \\
\hline & CIII 1175.8 & .3505 & -3 & - & & .3378 & +2 \\
\hline & CIV 1550.8 & .5581 & -3 & - & & .1280 & +5 \\
\hline & cont. 1458.0 & .4996 & -3 & - & & .1016 & -2 \\
\hline & $\mathrm{H} \gamma \quad 4340.5$ & .1776 & +2 & - & & .3061 & -0 \\
\hline & cont. 4340.5 & .2696 & -2 & - & & .1022 & -2 \\
\hline \multirow[t]{6}{*}{0.01} & $\begin{array}{ll}\text { CII }_{1} \quad 1335.3\end{array}$ & .1643 & +6 & .1814 & +6 & .2040 & +2 \\
\hline & CIII 1175.8 & .3249 & -2 & .1046 & +3 & .6355 & +3 \\
\hline & Culv 1550.8 & .5487 & -2 & .2714 & -1 & .1170 & +6 \\
\hline & cont. 1458.0 & .4848 & -2 & .6508 & -2 & .8764 & -2 \\
\hline & $\mathrm{H} \gamma \quad 4340.5$ & .1214 & +3 & .3768 & +2 & .3668 & +1 \\
\hline & cont. 4340.5 & .2899 & -1 & .1066 & -1 & .9739 & -2 \\
\hline \multirow[t]{6}{*}{0.10} & CII 1335.3 & .8476 & +6 & .4913 & +6 & .2804 & +3 \\
\hline & CIII 1175.8 & .2861 & -1 & .2719 & +4 & .5784 & +4 \\
\hline & CIV 1550.8 & .5153 & -1 & .5284 & +2 & .7578 & +6 \\
\hline & cont. 1458.0 & .4471 & -1 & .7207 & -1 & .7161 & -1 \\
\hline & $\mathrm{H} \gamma \quad 4340.5$ & .5194 & +3 & .2170 & +3 & .4623 & +2 \\
\hline & cont. 4340.5 & .4590 & +0 & .1586 & -0 & .1206 & -0 \\
\hline \multirow[t]{6}{*}{0.50} & CII 1335.3 & .3054 & +7 & .6833 & +6 & .5045 & +3 \\
\hline & CIII 1175.8 & .4428 & +1 & .1234 & +5 & .1111 & +5 \\
\hline & CIV 1550.8 & .2801 & -0 & .1782 & +5 & .2853 & +7 \\
\hline & cont. 1458.0 & .2452 & -0 & .3799 & -0 & .3289 & -0 \\
\hline & $\mathrm{H} \gamma \quad 4340.5$ & .1426 & +4 & .6216 & +3 & .1898 & +3 \\
\hline & cont. 4340.5 & .4377 & +1 & .1097 & +1 & .8028 & +0 \\
\hline
\end{tabular}

a Represented by model P13, see Underhill (1968b).

b Represented by model B13, see Guillaume (1966).

c Represented by model PPB40, see Underhill (1968a).

tinuous spectrum is concerned. Line blanketing has been taken into account for the B1.5 and O9 models. The monochromatic optical depths have been calculated from an assumed fractional abundance of carbon by weight of $3.25 \times 10^{-3}$; the fractional abundance of hydrogen is 0.68 , that of helium is 0.32 . The degree of ionization and excitation has been calculated by the Saha and Boltzmann laws.

In all three models and at all levels considered, the monochromatic optical depth at the centre of $\mathrm{H} \gamma$ is at least 100 times that in the continuum at the same wavelength. This ratio is typical for strong lines in the normally observed spectral region. Since models are constructed to represent the continuous spectrum well in the normally observed spectrum region, they usually are made in insufficient detail to permit one to calculate the centre of $\mathbf{H} \gamma$ accurately. This is certainly true for late B type models. Furthermore in the outermost layers of models the electron density is sufficiently low, usually less than $10^{12}$, that the assumption of the Saha and Boltzmann laws is of doubtful validity. 
In the ultraviolet region the continuous absorption coefficient is small with the result that layers near or deeper than $\tau=0.5$ are most significant for forming the continuous spectrum. On the other hand, in the centres of resonance lines the monochromatic opacity may become very large, some $10^{6}-10^{8}$ times that in the neighbouring continuous spectrum; in many cases the model may be opaque in the line before one has traversed a significant opacity in any continuum frequency. In that case the model defined by properties of the continuous spectrum and so identified with a star is probably not at all relevant for predicting the profile of the absorption line. One is observing an entirely different source in the resonance line from what one is observing in the normally observed parts of the spectrum.

The numerical results shown in Table I, taken at their face value, suggest that the CII lines at $1335 \AA$ will be very strong in B6 stars, but the C III and CIV lines will be weak. At type B1.5 one expects all three groups of lines to be strong, but the $\mathrm{C}$ II lines will be stronger than $\mathrm{C}$ III or CIV. At type $\mathrm{O} 9$ the pattern will be reversed, the $\mathrm{C}$ IV lines being strongest. Such predictions are of little real meaning because (a) the models are not defined in sufficient detail to represent accurately the outermost layers of mainsequence B type stars, and (b) the adopted LTE theory is certainly not valid in the outermost layers of these stars, i.e. in the region where the resonance lines are formed.

When the ratio $l_{v} /\left(\kappa_{v}+l_{v}+\sigma\right)$ is very large, the hypothesis of plane parallel layers becomes of doubtful validity and one should take into account the fact that a star is a three-dimensional object. The observed radiation in the centre of a strong line will give information about physical conditions in an outer spherical shell which may have a radius significantly larger than that of the photosphere which produces the continuous radiation. If there is a differential field of motion in the extended spherical atmosphere, for instance a uniform expansion, the spectral lines will have a P Cygni shape consisting of an emission component flanked by a shortward displaced absorption component. Simple examples of typical P Cygni line profiles were first predicted by Beals $(1929,1934)$; recent applications of this type of geometric theory to interpreting the spectra of supergiants have been made by Hutchings (1968). In a purely geometric theory usually a very simple assumption is made about the source function at each part in the atmosphere; often this is that the source function is the Planck function at an assigned local temperature.

\section{The Physics of Line Formation}

For the following discussion the geometry of the model is plane parallel layers. It is assumed that the distribution of the atoms over their various possible stages of ionization and over the individual energy levels of each stage of ionization may be found from the requirement that statistical equilibrium exist. This constraint is represented by a set of simultaneous equations, one for each state of the atom, expressing the condition that the number of particles entering each energy level per unit time is equal to the number leaving. The processes which cause transfer of particles from one level to another a re collisions (chiefly with electrons), spontaneous emission of radiation, 
impressed emission, photoexcitation, photoionization and recombination. To obtain a quantitative measure of the effect of these processes, the collision and radiative rates must be known. These data are provided by atomic physics. The collision rates depend upon the density of particles; some of the radiative rates depend upon the photon density.

In principle each atom and ion possesses an infinite number of levels; in practice the number of levels is limited by Stark broadening and the consequent disappearance of high levels owing to perturbations by the surrounding electrons and ions. In addition, the number of levels which must be considered separately can be restricted by realising that levels close to the continuum will have Boltzmann populations relative to the continuum population owing to the dominance of collisional processes between these high-lying levels. The art of handling the radiative transfer process in stellar atmospheres is to find out in the relevent situation to how low a level one may use the Boltzmann law without seriously misrepresenting the physics of spectral line formation. Since the Boltzmann law is an explicit formula which depends only on the local electron temperature, its use simplifies the calculation of level populations.

The transfer of radiation of frequency $v$ in a stellar atmosphere consisting of plane parallel layers may be represented by the equation

$$
\frac{\mu \mathrm{d} I(t, \vartheta, v)}{\mathrm{d} t}=\frac{\left(\kappa_{v}+l_{0} \varphi_{v}+\sigma\right)}{\kappa} I(t, \vartheta, v)-S(t) .
$$

Here the monochromatic line absorption coefficient has been written as $l_{0} \varphi_{v}$ where $l_{0}$ is an appropriate combination of physical constants and the population, $n_{1}$, of the energy level (denoted by the subscript 1 ) from which the absorption line arises and $\varphi_{v}$ is a normalised shape function.

Thus

$$
\int_{0}^{\infty} \varphi_{\nu} \mathrm{d} v \equiv 1
$$

at each point in the atmosphere. In general $\varphi_{v}$ is a function of the local electron temperature and density. A characteristic opacity coefficient $\kappa$ has been selected to define a characteristic optical depth, $t$, by which to measure geometric depth in the atmosphere.

By definition, the source function $S(t)$ is the ratio of the emissivity at frequency $v$ to the characteristic opacity coefficient used to define the depth scale. The line source function is found by considering in detail what can happen to a photon which is absorbed and excites an atom from the lower level of the transition being considered, to level 2, the upper level of the line.

It is not appropriate here to go deeply into the details of the theory for the resonance and other intrinsically strong lines which occur in the ultraviolet spectral region. These problems of physics and of numerical analysis have engaged the attention of a number of workers in recent years. The notation of Hummer $(1962,1964,1968)$ and of Avrett and Hummer (1965) will be followed here, with the change that the quantity they call $\varepsilon$ 
will be called $\lambda$ in order to reduce confusion with a related quantity called $\varepsilon$ by Thomas (1957, 1965) and by Jefferies and Thomas (1958). According to Avrett and Hummer it is useful to define a quantity $\lambda(\equiv \varepsilon)$ which gives the probability that a photon in line frequencies is absorbed and then disappears. The disappearance may be due to collisional de-excitation of the atom or to radiative de-excitation via another line. For resonance lines or for strong subordinate lines from low-lying levels when the lineformation process takes place in layers of the stellar atmosphere which are not particularly dense, the particle density being of the order of $10^{11}-10^{13}, \lambda$ may take values of the order of $10^{-4}-10^{-8}$.

Let us ignore, for simplicity, the continuous opacity $\kappa_{v}$ and the opacity due to electron scattering, $\sigma$. Then one can write the source function in line frequencies as

$$
S_{L}(t)=\{1-\lambda(t)\} \int_{0}^{\infty} \varphi_{v}(t) J_{v}(t) \mathrm{d} v+\lambda^{*}(t) B_{v}(t) .
$$

Here

$$
\mathrm{d} t=-l_{0} \varrho(z) \mathrm{d} z
$$

where $\varrho(z)$ is the density and $\lambda^{*}(t) B_{v}(t)$ is the contribution to the line source function arising from the excitation of atoms to level 2 by all other processes than photoexcitation from level 1. The second term on the right side of Equation (5) is written as a coefficient, $\lambda^{*}$, multiplied by the Planck function $B_{v}(t)$ in order to stress the formal resemblance between Equation (4) and the forms for the source function which are obtained when the line-formation problem is simplified by assuming either that the line-formation process is one of coherent, isotropic scattering or that it takes place as if local thermodynamic equilibrium, LTE, existed at each point in the atmosphere. In each case the Saha and Boltzmann equations are used to estimate the level populations.

The first term on the right side of Equation (4) represents non-coherent scattering with complete redistribution, while the second term is equivalent to using Kirchhoff's law to estimate the emissivity. Avrett and Hummer (1965) and Hummer (1968) have studied in detail how to solve a transfer equation in which the source function has a form like Equation (4) and they have given some results for isothermal finite atmospheres and for isothermal semi-infinite atmospheres. A result of importance for our discussion which may be extracted from their work is that in semi-infinite atmospheres strong absorption lines from low-lying or ground levels will have significantly deeper cores than would be predicted using the hypothesis of LTE, the number of atoms in level 1 being the same in both cases. Thus it may be expected that when an improved theory of line formation is used, stronger lines will be found than are found using the hypothesis of LTE.

In the case of line formation in a fairly dense atmosphere, the particle density being of the order of $10^{15}-10^{16}$, the theory of line formation sketched above goes over to the well known, simplified theory of line formation obtained when the hypothesis of LTE is made. Then $\lambda \rightarrow 1$ and $\lambda^{*} \rightarrow 1$. Whether one is justified in adopting a simple LTE theory or not depends upon the characteristics of the atom, that is upon the collision 
cross-sections for transfer to and from the levels of interest for the line under study and upon the radiative cross-sections as well as upon the particle density and the photon density in the atmosphere.

Very many of the strong lines in the ultraviolet spectral region come from low-lying, rather isolated levels. Because the lines are intrinsically strong these lines are formed high in the atmosphere where the particle density is low. Consequently one may expect that if one is to obtain a meaningful interpretation of the spectrum it will be necessary to use a rather general theory of line formation in which the restrictive hypothesis of LTE is not made.

The possibility of interpreting stellar line profiles using the hypothesis that line formation occurs as if isotropic, coherent scattering occurred while the level populations are given by Saha's and Boltzmann's laws will not be considered, for these hypotheses are incompatible. Such a formulation of the problem of line formation leads to deeper lines, for the same number of atoms, than does the hypothesis of LTE.

The only predictions of ultraviolet stellar absorption lines available at present are those by Gaustad and Spitzer (1961), Morton (1965), Guillaume, Van Rensbergen and Underhill (1965), Guillaume (1966), Elst (1966), and Underhill (1968a). The work of Gaustad and Spitzer gives a schematic survey which is essentially replaced by the work of Morton who like Elst, Guillaume, Van Rensbergen and Underhill has used the hypothesis of LTE in the stellar atmosphere. This hypothesis can certainly not be justified for most of the lines studied, but the results give some idea of what sort of absorption lines may be expected in the ultraviolet spectrum of a main-sequence star of type B. No similar studies have yet been made of supergiant atmospheres and none are likely to be made because it is thoroughly understood that the restrictive hypothesis of line formation in LTE in plane parallel static atmospheres is unsuited to supergiants. The LTE computations have shown that the blanketing by strong lines is significant at wavelengths shorter than $1900 \AA$ (Morton, 1965) while at wavelengths between 1900 and $3000 \AA$ it is of the order of 0.1 mag per $100 \AA$ (Elst, 1966).

\section{The Spectroscopic Description of Some Typical Ultraviolet Multiplets}

Partial, scaled energy-level diagrams are shown in Figure 1 for some of the ions that have strong lines in the far ultraviolet. Lines have been drawn indicating the most conspicuous multiplets. The spectroscopic notation and wavelengths of these multiplets are given in Table II. Profiles of many of these lines have been predicted by Morton (1965) using a model atmosphere representing stars of about type B1.5V. Morton's calculations were done assuming that the level populations may be found using the Saha and Boltzmann laws.

Two important conclusions can be drawn from Figure 1:

(1) The energy levels between which most of the strong ultraviolet lines occur are relatively isolated. That is their separation in energy from each other is several times greater than the average energy of electrons at temperatures near $15000^{\circ}$ which is a typical value for the outer layers of an early B type star. 


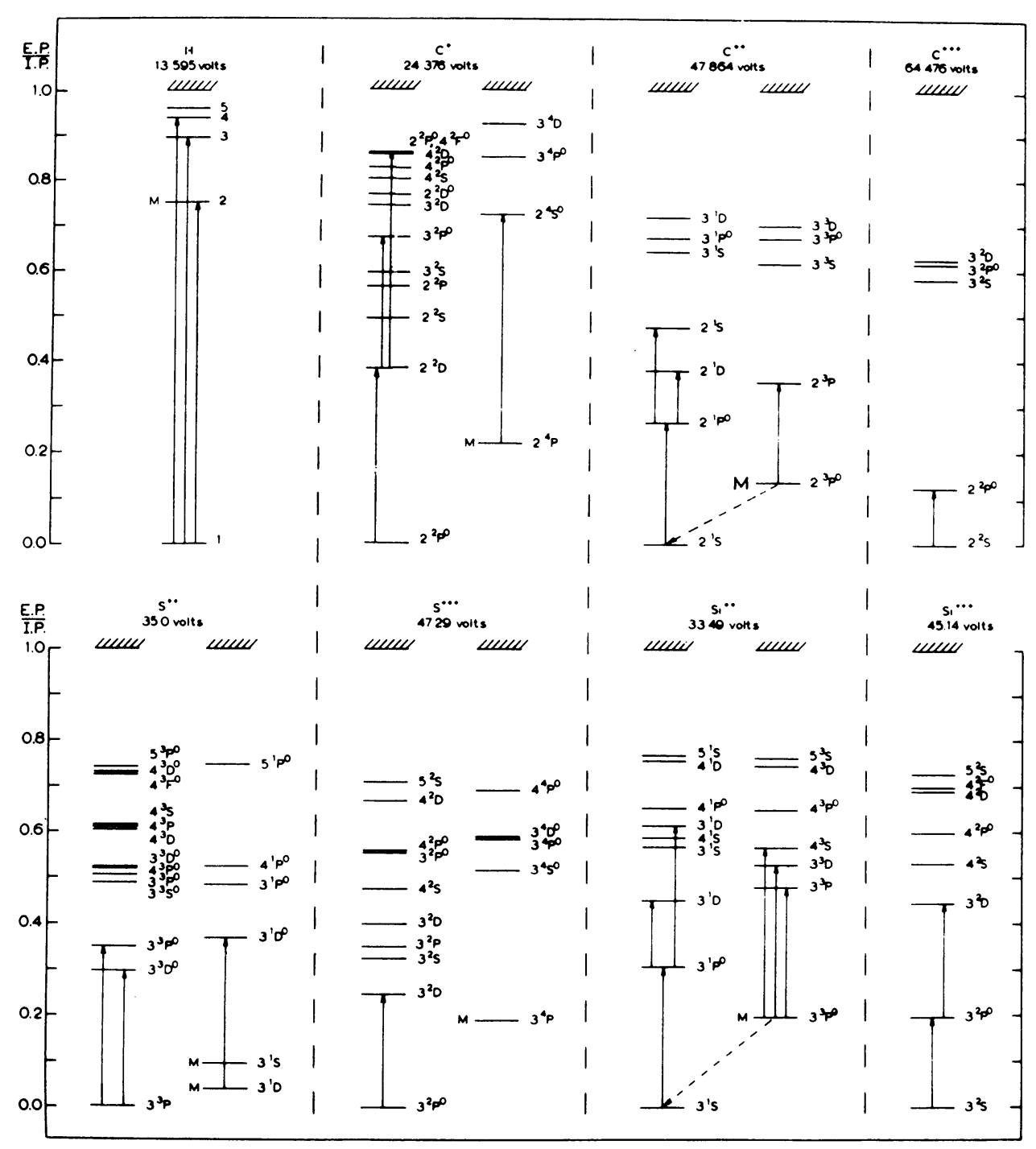

Fig. 1. Partial, scaled energy-level diagrams. More levels exist above the highest level drawn but no known levels have been left out at lower energies. The primary ionization energy is indicated below the symbol for each ion. Strong ultraviolet multiplets are represented by vertical arrows. Intersystem lines are represented by broken lines.

(2) Metastable levels occur in many of the energy-level schemes and intrinsically strong lines are observed from these levels.

These facts indicate that the populations of the levels from which the strong ultraviolet lines come should be found by solving the equations of statistical equilibrium. Boltzmann populations relative to the ground level will only be obtained if collisional excitation predominates or if there is detailed radiative balance in the line. It is not 
TABLE II

Ultraviolet lines and multiplets shown in Figure 2

\begin{tabular}{|c|c|c|c|c|c|}
\hline Spectrum & $\lambda_{\text {vac }}(\AA)$ & Transition & Spectrun & $\lambda_{c a c}(\AA)$ & Transition \\
\hline $\mathbf{H}$ & 1215.7 & -2 & SilII & $1206.51^{\mathrm{b}}$ & $3^{1} \mathrm{~S}-3^{1} \mathrm{P}^{\mathrm{o}}$ \\
\hline $\mathbf{H}$ & 1025.7 & -3 & Si IIII & 2541.8 & $3^{1} \mathrm{P}^{0}-3^{1} \mathrm{D}$ \\
\hline $\mathbf{H}$ & 972.5 & -4 & SillI & 1417.2 & $3^{1} \mathbf{P}^{0}-3^{1} \mathrm{~S}$ \\
\hline $\mathrm{C}_{\text {II }}$ & $1334.5,1335.7$ & $2^{2} P^{o}-2^{2} D$ & SillI & 1300 & $3^{3} P^{o}-3^{3} P$ \\
\hline CII & $1760.4,1760.8$ & $2^{2} \mathrm{D}-3^{2} \mathrm{Po}$ & SilII & 1110 & $3^{3} P^{0}-3^{3} D$ \\
\hline CII & $1065.9,1066.1$ & $2^{2} \mathrm{D}-2^{2} \mathrm{P}^{o}$ & SiIII & 996 & $3^{3} P^{0}-4^{3} S$ \\
\hline CII & 1010 & $2^{4} P-2^{4} S^{0}$ & [Si III] & 1892.0 & $3^{1} S-3^{3} P^{o}$ \\
\hline CIII & 977.0 & $2^{1} S-2^{1} P^{o}$ & Silv & $1393.8,1402.8$ & $3^{2} S-3^{2} P^{o}$ \\
\hline CIII & $2296.9^{a}$ & $2^{1} P^{o}-2^{1} D$ & Silv & 1128 & $3^{2} \mathrm{P}^{0}-3^{2} \mathrm{D}$ \\
\hline CIII & 1247.4 & $2^{1} P^{0}-2^{1} S$ & SIII & 1194 & $3^{3} P-3^{3} D^{c}$ \\
\hline $\mathrm{C}_{\text {III }}$ & 1176 & $2^{3} P^{0}-2^{3} P$ & SIII & 1015 & $3^{3} P-3^{3} P^{0}$ \\
\hline [CIII] & 1909 & $2^{1} S-2^{3} P^{o}$ & SIII & 1077.8 & $3^{1} \mathrm{D}-3^{1} \mathrm{D}^{\mathrm{c}}$ \\
\hline Civ & $1548.2,1550.8$ & $2^{2} S-2^{2} \mathbf{P}^{0}$ & SIV & 1072 & $3^{2} P^{0}-3^{2} D$ \\
\hline
\end{tabular}

a Wavelength in air.

b Will be observed blended with $1206.533^{1} \mathrm{Po}_{-} 3^{1} \mathrm{D}$.

very probable that either of these conditions is satisfied in the extreme outer atmospheres of B stars.

The C III intersystem line at $1909 \AA\left(2{ }^{1} \mathrm{~S}_{0}-2{ }^{3} \mathrm{P}_{1}^{\mathrm{o}}\right)$ has been observed in emission in the spectrum of the Wolf-Rayet binary star $\gamma_{2}$ Velorum (Stecher, 1968). It would be interesting to look for the equivalent $\mathrm{Si}$ III intersystem line at $1892 \AA\left(3^{1} \mathrm{~S}_{0}-3^{3} \mathrm{P}_{1}^{\mathrm{o}}\right)$ in stars with extended atmospheres having not too high an electron temperature.

\section{Discussion}

The study of line profiles obtained from high-resolution ultraviolet spectra of stars should give information about the physical conditions in the outermost layers of stars, but meaningful information will not be obtained unless the analysis is done using a sufficiently general theory of line formation. Such theories are being developed and they can be tested using profiles of resonance lines and strong lines from low-lying levels which occur in the spectral region accessible from the surface of the earth. It is to be hoped that adequate theories will be available by the time adequate profiles of the strong ultraviolet lines have been obtained.

Because the shapes and depths of the cores of resonance lines and of strong lines from low-lying levels are sensitive to the density and geometric extent of the atmosphere, the strong ultraviolet lines may have rather different strengths in B stars having similar spectra in the normally observed region. Although the lines used for spectral classification are strong, and consequently somewhat sensitive to the density and extent of the atmosphere, they are not expected to be as sensitive as the far ultraviolet lines. It is rather probable that stars having the same spectrum in the $3900-5000 \AA$ region will not have identical ultraviolet spectra. If this speculation is proved true by obser- 
vation, then it may be rather difficult to obtain a reliable estimate of the shape of the interstellar extinction law in the far ultraviolet.

Many B type stars rotate rapidly, the rotation producing broad shallow absorption lines. Since the broadening or displacement of spectral lines by motion varies as $v \lambda / c$, where $v$ is the velocity causing the broadening or displacement, $\lambda$ is the wavelength, and $c$ is the velocity of light, the broadening or shift of the ultraviolet lines will be reduced by a factor between 2 and 3 from that of lines in the normally observed spectral region. Consequently, unless observations are obtained with a resolution better than about $0.2 \AA$, only the most rapidly rotating stars will show the well known dishshaped lines. Similarly it will only be possible to separate the spectra of double-lined spectroscopic binaries in the ultraviolet when the velocity difference is large. Rather high effective resolution (projected slit width in the spectrum) will be required to obtain true profiles of ultraviolet lines, particularly of the core of the line which is the part most sensitive to the physical conditions in the atmosphere.

\section{References}

Avrett, E. H. and Hummer, D. G.: 1965, Monthly Notices Roy. Astron. Soc. 130, 295.

Beals, C. S.: 1929, Monthly Notices Roy. Astron. Soc. 90, 202.

Beals, C. S.: 1934, Publ. Dom. Astrophys. Obs. Victoria 6, 93.

Elst, E. W.: 1966, Bull. Astron. Inst. Netherl. 19, 90.

Gaustad, J. E. and Spitzer, L.: 1961, Astrophys. J. 134, 771.

Guillaume, C.: 1966, Bull. Astron. Inst. Netherl.18, 175.

Guillaume, C., Van Rensbergen, W. and Underhill, A. B.: 1965, Bull. Astron. Inst. Netherl. 18, 106.

Hummer, D. G.: 1962, Monthly Notices Roy. Astron. Soc. 125, 21.

Hummer, D. G.: 1964, Astrophys. J. 140, 276.

Hummer, D. G.: 1968, Monthly Notices Roy. Astron. Soc. 138, 73.

Hutchings, J. B.: 1968, Monthly Notices Roy. Astron. Soc. 141, 329.

Jefferies, J. T. and Thomas, R. N.: 1958, Astrophys. J. 127, 667.

Morton, D. C.: 1965, Astrophys. J. 141, 73.

Stecher, T. P.: 1968, in Wolf-Rayet Stars (ed. by K. B. Gebbie and R. N. Thomas), N.B.S. Special Pub. 307, p. 65.

Thomas, R. N.: 1957, Astrophys. J. 125, 260.

Thomas, R. N.: 1965, Some Aspects of Non-Equilibrium Thermodynamics in the Presence of a Radiution Field, University of Colorado Press, Boulder, Colo. A full set of references to work in this field is found here.

Underhill, A. B.: 1968a, Bull. Astron. Inst. Netherl. 19, 500.

Underhill, A. B.: 1968b, Bull. Astron. Inst. Netherl. 19, 526.

\section{Discussion}

Gingerich: Underhill's remarks on non-LTE seem convincing for cores of lines, and they suggest that if we are only interested in UV resonance lines in terms of opacity and line blocking, we can calculate models with lines represented by rectangles of infinite opacity. On the other hand, the wings can be formed deep enough to be in LTE; since we need to know the width of the approximating rectangle, what we really need are damping parameters. Note that in using $\mathrm{H} \gamma$ profiles to determine the gravity of A stars, we fit only the wings and ignore the core, so LTE still works satisfactorily.

Underhill: The critical question is how far out in the wings must you be before the LTE hypothesis becomes tenable. I suspect that in the ultraviolet you will by this time have run into another line which is very strong. Fortunately this does not occur at $\mathrm{H} \gamma$ in A stars. 
Hekela: There are some misunderstandings in the description of atmospheric structure and the following interpretation of lines. But the problem is not LTE or non-LTE, because both are methods dealing directly only with radiative processes. The question is to discover such a set of characteristic equations that include all radiative and dynamical processes. Such a set would be different for different astronomical objects. This is the only way, though, I am sorry, an extremely complicated one, by which it would be possible to overcome continuously accumulated discrepancies in astrophysics.

Further, from the theoretical standpoint, it is not desirable to observe mostly only equivalent widths, because of the small amount of physical information in them.

I hope recent developments of detection techniques will allow us to measure line profiles as a function of position on the disc for many objects. If it would be possible to observe some of them by methods of absolute spectrophotometry we would obtain excellent input for a set of characteristic equations.

Underhill: The question of LTE or non-LTE is not only one of radiation processes for one must take into account collisional excitations and deexcitations as well as radiative processes. Furthermore, the line shape and position of the line centre comes in. In the most general formulation the dependence of these on a velocity field can be worked into the set of equations for statistical equilibrium and for radiative transfer. Solution of such a coupled set of equations would be extremely difficult and it is certainly a problem that should be looked at in more detail than has yet been done. The dynamic flow (or lack of it) must be used to determine the pressure structure in an atmosphere. The radiation flow determines, in principle, the temperature structure. These are related through the perfect gas law. 